\title{
Obrigkeitliche Maßnahmen gegen die Pest in Stadt und Herrschaft Rheinfelden im 16. und 17. Jahrhundert
}

Von Vera Waldis

\section{Einleitung}

Die Geschichte der Pest weist mit den verschiedensten Gebieten Berührungspunkte auf:

Individuelle psychologische Reaktionen, sozial- und bevölkerungsgeschichtliche Faktoren spielen dabei ebenso ihre Rolle wie die medizinhistorisch wichtigen Entwicklungen auf dem Gebiete der prophylaktischen Seuchenbekämpfung und dem damit Hand in Hand gehenden Aufbau des öffentlichen Gesundheitswesens.

In der ehemals vorderösterreichischen Stadt und Herrschaft Rheinfelden wurde die Pestprophylaxe im Laufe des 16. und 17. Jahrhunderts aufgebaut. Die Verantwortlichen trafen ihre Maßnahmen aufgrund ihrer ätiologischen Vorstellungen. Wie sich diese im Laufe der Zeit änderten, spiegelt sich in den für die «Pest» verwendeten Begriffen wider. Eine Änderung des Begriffs zog eine neue Maßnahme nach sich. Für deren Durchsetzung waren die verwaltungsrechtlichen Kompetenzen ausschlaggebend, insbesondere, da die prophylaktischen Maßnahmen für den Handel äußerst einschneidend und somit unwillkommen waren.

\section{Die Verwaltung von Stadt und Herrschaft Rheinfelden im 16. und 17. Jahrhundert}

Als ehemals freie Reichsstadt hatte Rheinfelden auch noch unter österreichischer Herrschaft (seit 1449) gewisse Privilegien gegenüber andern Habsburger Städten ${ }^{1}$. Es galt als eigener Verwaltungsbezirk und besaß die Rechte der niederen und hohen Gerichtsbarkeit. Zusammen mit den andern drei Waldstädten am Rhein, Waldshut, Laufenburg und Säckingen, unterstand Rheinfelden dem Hauptmann der vier Waldstädte ${ }^{2}$. Dieser hatte aber, was die Stadtverwaltung im Innern anbelangte, nur beratende Funktion ${ }^{3}$. Gemeinsame Angelegenheiten der vier Waldstädte wurden jeweils am «Städtetag» in Laufenburg behandelt ${ }^{4}$, wo auch die Befehle des Hauptmanns und die der österreichischen Regierung verlesen wurden ${ }^{5}$. An der Spitze der städtischen Verwaltung Rheinfeldens stand der Schultheiß, welcher gemeinsam mit dem Täglichen Rat und bei wichtigeren Geschäften auch mit dem Großen Rat die Stadtordnungen erließ. Beide Räte bestanden nur aus Zunftmitgliedern. Im 15. Jahrhundert nannte sich der Kleine 
Rat noch «rat mit vier», das bedeutete, daß aus jeder der drei Zünfte vier Männer in diesem Gremium saßen. Manchmal wurden von diesen die sechs Mitglieder jedes Zunftvorstandes beigezogen, so daß dann die gesetzgebende Behörde aus dem Schultheißen und 30 Räten bestand. Daraus entwickelte sich später der Große Rat. Hin und wieder wurde die ganze Bürgergemeinde einberufen, welche aber kaum noch Entscheidungsgewalt hatte; meist wurde ihr nur noch das Ergebnis der vorangegangenen Beratung mitgeteilt ${ }^{6}$.

Getrennt von der Stadt wurde das umliegende Landgebiet, die Herrschaft Rheinfelden, von einem k. k. Oberamt unter der Leitung des Oberamtmanns verwaltet $^{7}$. Das Gebiet dieser Kameralherrschaft erstreckte sich über die Landschaft Rheintal, auf dem rechten Ufer des Rheins, und die zwei linksufrigen Landschaften Möhlinbach und Fricktal ${ }^{8}$ (vgl. Kärtchen S. 208).

Bis 1798 waren Stadt und Herrschaft Rheinfelden dem vorderösterreichischen Breisgau angegliedert und unterstanden somit der k. k. Regierung und Kammer der österreichischen Vorlande. Diese hatte bis zum Ende des Dreißigjährigen Krieges ihren Sitz im elsässischen Ensisheim, nach 1651 in Freiburg i.Br. Ihrerseits unterstand sie der oberösterreichischen Regierung, welche in Innsbruck residierte ${ }^{9}$.

\section{Rheinfelden als Transitgebiet}

Obwohl Rheinfelden zu Österreich gehörte, spielte es durch seine Lage am Oberrhein eine gewisse Rolle in der Pestgeschichte der Schweiz; denn die Pestgeschichte der Schweiz ist unzertrennlich mit deren Handelsgeschichte verflochten ${ }^{10}$.

Es wäre deshalb wichtig, die Handelsstraßen der Schweiz im Detail zu kennen; doch lassen sie sich vorläufig nur über größere Strecken hinweg skizzieren:

Von Mailand aus ging der Handelsverkehr entweder über den Gotthard oder die Bündnerpässe in Richtung der Hafenstädte an der Nordsee und nach Amsterdam. Im schweizerischen Mittelland wurden die Handelsgüter auf dem Wasserweg transportiert (Vierwaldstättersee-Reuß-Aare oder Walensee-ZürichseeLimmat-Aare $)^{11}$. In welchem Maße sich die Niederschiffahrt auch auf den Rhein unterhalb der Aaremündung ausdehnte, ist nicht sicher, da der Rhein von hier bis Rheinfelden einige schwer schiffbare Stellen aufwies - vor allem die Stromschnellen («Laufen») kurz nach der Aaremündung und die bei Laufenburg ${ }^{12}$. Jedenfalls wurde es Reisenden von Innsbruck nach Ensisheim empfohlen, zwischen Schaffhausen und Rheinfelden den Landweg über Waldshut und Säckingen zu nehmen ${ }^{13}$. Auf dieser Strecke war ja auch der Rheinfall bei Laufen ein unüber- 
windliches Hindernis für die Schiffahrt. Zwischenstation für alle diese Routen war aber immer Basel. In umgekehrter Richtung kam nur der Landweg in Frage. Der Gotthardverkehr ging deshalb von Basel in der Hauptsache nicht mehr durch österreichisches Gebiet, sondern über den Hauenstein nach Olten und von da nach Luzern weiter. Doch die Bündnerpässe wurden dank der Bözbergstraße noch immer über Rheinfelden und Zürich erreicht ${ }^{14}$.

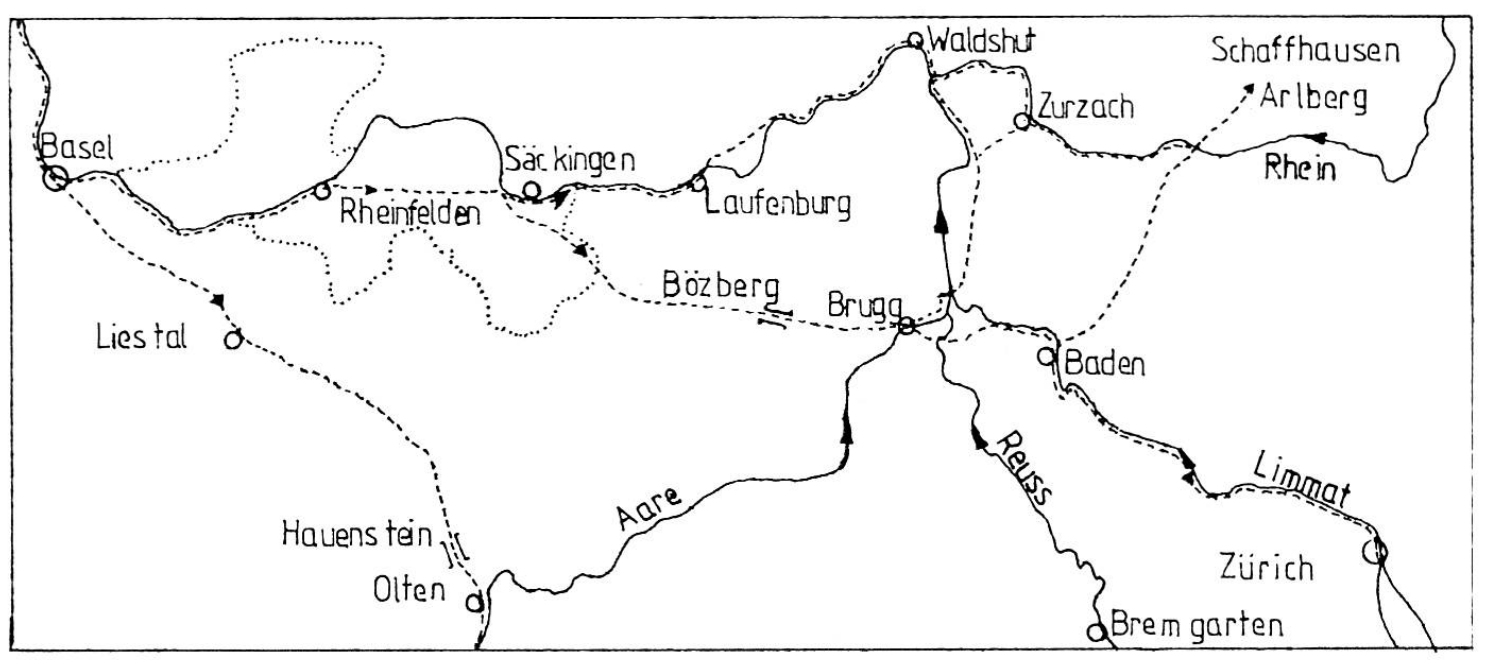

Übersichtskarte: Die Haupthandelswege durch und um Rheinfelden ${ }^{17}$

Aber auch in ost-westlicher Richtung gab es einen gewissen Güteraustausch, dessen Weg durch rheinfeldisches Gebiet führte: Schon im Mittelalter wurde das Salz für die österreichischen Vorlande von Hall im Inntal über den Arlberg eingeführt. Dieser Weg wurde auch in umgekehrter Richtung benutzt, um Waren aus Deutschland, aber auch aus der Schweiz auf die Haller und Bozener Märkte zu bringen ${ }^{15}$. Es scheint somit, daß die Arlberg-Route als eine Zweigstraße der sogenannten «Venedigerstraße», welche über den Brenner führte, betrachtet werden muß. Diese Überlegung würde auch noch besser erklären, weshalb Zurzach zu seiner großen Bedeutung als Messeort kam ${ }^{16}$.

Während der Pestepidemien waren die zwei Handelsumschlagplätze Basel und Zurzach immer am meisten gefährdet. Auch Rheinfelden wurde dadurch, vor allem von Basel her, ständig bedroht. Die Rheinfelder hatten nämlich auch mannigfaltige persönliche Beziehungen zu der nahe gelegenen Stadt Basel. 
3. Die Grundlagen zur Entwicklung obrigkeitlicher Maßnahmen gegen die Pest in Stadt und Herrschaft Rheinfelden im 16. und 17. Jahrhundert

In der vorderösterreichischen Herrschaft Rheinfelden wurden die wirksamen prophylaktischen Maßnahmen im Laufe des 17. Jahrhunderts entwickelt. Es war das Verdienst des Oberamtmanns Johann Christoph Hug ${ }^{18}$ (Doktor beider Rechte und vorderösterreichischer Regierungsrat), diese Maßnahmen im Pestzug von 1667 bis 1669 erstmals in vollem Umfang angeordnet zu haben. Mit Hilfe von Landschreiber und Einnehmer Ferdinand Sehler und dessen Nachfolger Marx Jacob Beltz (ungefähr ab Dezember 1668) führte Hug eine ausführliche Korrespondenz über die «Contagion» und sandte dann, den jeweiligen Umständen entsprechend, seine Befehle an die drei Landschaften der Herrschaft ${ }^{19}$. Die Maßnahmen lassen sich mit den zwei ursprünglich aus Italien stammenden Begriffen «Bando» und «Suspension» definieren, die die Einreise (oder Durchreise) von Personen und die Einfuhr beziehungsweise den Transit von Handelsgütern regelten. Wurde der Bando über ein von der Pest verseuchtes Gebiet verhängt, durften weder Personen noch Waren von dort eingelassen werden. Über Gebiete, die im Verdacht standen, daß Pestfälle aufgetreten waren, wurde die Suspension verhängt. Dies bedeutete, daß Personen oder Waren aus diesen Gebieten zuerst an der Grenze für eine gewisse Zeit isoliert wurden, bis sich der Verdacht als falsch erwies, oder auch, bis es sicher war, daß die so isolierten Reisenden und Kaufleute, nach längerer Quarantäne, nicht mehr an der Pest erkranken konnten ${ }^{20}$. Diese differenzierten, spezifisch gegen die Pest gerichteten Abwehrmaßnahmen waren aber nur möglich, wenn die Vorstellungen über den Infektionsmodus dem tatsächlichen, komplexen epidemiologischen Geschehen einigermaßen entsprachen.

Erstmals hatte Gerolamo Fracastoro (etwa 1478-1553) eine Theorie aufgestellt, die der Pestübertragungsart, wie wir sie heute kennen, gerecht wurde (1546). Er lehrte, daß die Pest durch ein Kontagium übertragen werden konnte, und zwar nicht nur durch unmittelbaren Kontakt (per contactum), sondern auch durch Zwischenträger (per fomites) oder, als dritte Möglichkeit, über eine gewisse Entfernung hinweg [durch die Luft] (ad distans) ${ }^{21}$. Andere Gelehrte faßten den Begriff der Kontagiosität enger: Nach ihnen konnte die Übertragung nur durch direkte Berührung mit dem Pestgift zustande kommen. Wieder andere, von der antiken Tradition herkommend, nahmen an, daß ein Pestgift infolge unhygienischer Verhältnisse in die Luft abgedunstet werde und diese vergifte (MiasmaLehre), womit vor allem auch die zeitliche und örtliche Häufung der Todesfälle erklärt war. Doch konnte auch die Luft an sich verdorben sein, ohne daß sie von 
solchem Pestgift durchsetzt gewesen wäre. Ungünstige Planetenkonstellationen, klimatische Verschlechterungen, Katastrophen wie Erdbeben wurden ebenfalls als Grund für die Entstehung einer Pestepidemie angenommen. Oft stand hinter diesen Ursachen letztendlich der Wille Gottes, sei es als Ausdruck seiner unergründlichen Allmacht oder seines Zorns ${ }^{22}$ oder, wie bei Felix Platter (1536-1614), zur Regulierung der Bevölkerungszahl ${ }^{23}$.

Beinahe alle diese Auffassungen finden sich auch in den nichtmedizinischen Akten Rheinfeldens über die Pest. Und entsprechend der jeweils vorherrschenden Meinung wurden die Abwehrmaßnahmen gestaltet. Allerdings wurden zur Umschreibung dieser Ursachen nicht die gelehrten Ausdrücke verwendet, doch lassen sich die volkstümlichen Begriffe ohne weiteres mit den Fachausdrücken korrelieren:

Allgemein üblich schon im Mittelalter war der Ausdruck «Sterbet» / «Sterbent» für die Pest. Als Verb kommt dieser Ausdruck auch in einer Stiftungsurkunde Rheinfeldens von 1420 vor. Es handelt sich um eine Stiftung, die dem Fridolinsaltar zu St.Martin, «vor etwas zites als es by uns mit sterben übel gienge $»,{ }^{24}$ gemacht wurde: Er steht als Beschreibung für das unfaßbare allgemeine Umkommen und gibt keinerlei ätiologische Hinweise. Diesem Dahinsterben vorbeugen zu wollen, war sicherlich hoffnungslos.

In Rheinfelden wurde die Pest erst 1564 wieder erwähnt ${ }^{25}$, als der Große Rat der Stadt zur Regelung der Kosten eine

«Ordnung jm Spittal dißer Sterbenden leiff halben»

aufstellte. Diese «Sterbenden leiff», später auch «grassierende Sterbeleüff», bringen den neuen Aspekt, daß die Pest wandert und sich ausbreitet.

Gleichzeitig wurde aber im ersten Absatz der Ordnung darauf hingewiesen, daß diese Krankheit göttlichen Ursprungs sei:

«Erstlichen, wafern, gott der allmechtig einen burger oder burgerin jnn disenn gefarlichen Sterbenndenn leuffenn, nach seinem göttlichen willen, Angriffenn, der oder die selbigenn, jre Krankhenn es sye Knecht oder magt jnn Spittal darjnnen zeerhaldten schicken wurden, ... ${ }^{26}$

Auch diese Vorstellungen konnten wohl kaum zu irgendwelchen prophylaktischen Maßnahmen Anstoß geben, gleich wie der begriff «böß luft» (vgl. S. 212), der $1582^{27}$ im Ratsprotokoll verwendet wurde, nicht zu einer sinnvollen Vorbeugung gegen die Pest führen konnte. Diese böse Luft scheint ihre Ursachen eher in ungünstigen kosmischen Bedingungen gehabt zu haben, wie sie schon von Aristoteles als luftverderbende Ursache überliefert ist ${ }^{28}$. 
Während des Pestzuges von 1582 scheinen einzelne Verantwortliche in Rheinfelden erstmals kontagionistische Ideen gehabt zu haben. So der Vogt der vier Waldstädte und Oberamtmann der Herrschaften Rheinfelden und Laufenburg, Hans Othmar von Schönau ${ }^{29}$. Er hatte verlangt, daß der Jahrmarkt in Rheinfelden wegen der Pest nicht abgehalten werden solle:

«Herr Schultheiß Brombach vnd Vngelter ${ }^{30}$ zeigen an das der Junckher Hans Othmar von Schönauw nach Jnen geschickht vnd begert nach dem allent halben sterbeleüff eingerissen, das man den künfftigen Jarmärckht abschaffen, vnd sonst pesser ordnung aufstellen, darauff einen Ersamen Rath weil es ein durchgeende straß hab, nit für rätlich ansicht. Doch wölle man sich darüber pesser vnd weiter bedenckhen, ...» ${ }^{31}$

Dieser Jahrmarkt wurde jedoch nach Beschluß des Großen Rates tatsächlich abgehalten $^{32}$, was zeigt, daß die Stadt eine gewisse Autonomie in ihrer Verwaltung hatte. Triebfeder für diesen Entscheid war der finanzielle Vorteil, welcher der Stadt durch den Jahrmarkt entstand. Es war bekannt geworden, daß der Basler Martinimarkt wegen der dort herrschenden Pest sehr schwach besucht worden war, was dem offenbar etwas später angesetzten Rheinfelder Jahrmarkt zugute kam:

«Jahrmarckhts halben ist erkandt, weil es wegen des sterbendts zu Basel daselbst ein kleine Meß gewesen, allhie ein großen Jarmerckt geben werde dz man den selben versehen sol aus jeder Zunft 4 Mann in Harnist, 2 ohn Harnist, vnd jedes thor 2 Mann dem Portner zuzegeben vnd .2. in das vßer heüßlin vffs Reinthor zu verordnen. ${ }^{33}$

Aus jeder der drei Zünfte ${ }^{34}$ wurden also 10 Wachen beordert, die während des Jahrmarktes für Recht und Ordnung zu sorgen hatten. Daß sie auch zur Kontrolle von Personen aus pestverseuchten Gegenden eingesetzt worden wären, läßt sich für diesen Zeitpunkt noch nicht nachweisen. Solche Maßnahmen gelangten erst später zur Anwendung.

Hingegen erschien es den Stadtvätern Rheinfeldens ganz praktisch, Vorsichtsmaßnahmen gegen die Pest zu verfügen, wenn damit der Handel nicht eingeschränkt wurde und es im Gegenteil darum ging, möglichst gute Bedingungen dafür zu schaffen: Es war in Rheinfelden üblich, daß am Allerseelentag [2. November] bedürftige Personen von auswärts in die Stadt kamen, wo sie von den Bürgern der Stadt ein Almosen erhielten. Die Infektionsgefahr gab nun Anlaß, daß diese nicht mehr ins Stadtzentrum eingelassen werden sollten, wo sie wahrscheinlich als störend empfunden wurden: 
«Armen Leuten halb so auf aller Seelentag alher kommen, ist erkandt, Weil die mereteils auß denen orten da es böß luft harkommen, das man die nit wie von altem her vmbgeen lassen, sonder in Spital behalte oder aber auf dem Kilchhof sitzen sollen, ...»

Bezeichnenderweise beschloß dies der Große Rat in der gleichen Sitzung, in welcher das Stattfinden des Jahrmarktes gutgeheißen wurde ${ }^{35}$.

Wäre es dem Rat wirklich nur darum gegangen, das Einschleppen des Pestgiftes durch die Bettler zu verhindern, hätte er konsequenterweise verfügen müssen, daß die Bettler und Armen schon an den Toren der Stadt abgewiesen worden wären. So aber war der direkte Kontakt zwischen Almosenempfänger und Spendern weiterhin gewährleistet, ebenso wie die Verbreitung des Pestgiftes durch die Luft.

Erst im folgenden Pestzug des Jahres 1593 sprach der Tägliche Rat der Stadt Rheinfelden von einer Infektion der Luft. Dies führte, wenn auch nur in ersten Ansätzen, zu Sperrmaßnahmen:

«... die Pettler vnd Armenleüth So von jnuizierten orthen khommen, sollen soueil müglich vnder den Thoren abgehalten vnd fortgewiesen werden. ${ }^{36}$

Wiederum waren es die Bettler, die davon am meisten betroffen waren. Doch scheinen auch vornehmere Bürger und Adelige sich vom öffentlichen Leben möglichst zurückgezogen zu haben: Seit 1541 war es in Rheinfelden üblich, daß der Schulmeister mit seinen Schülern ein Theaterstück inszenierte ${ }^{37} .1593$ wurde das schon geplante Spiel wegen der Pest vom Herbst auf den Frühling des folgenden Jahres verschoben:

«Schuolmeister Spils halben, ist einhellig erkhanndt, weyl leider der Luft an etlichen orten vmb die Statt jnuiciert. derenwegen die herren vnd vom Adel nit alher khomen, die kleidlungen [Kostüme] übell zue bekhommen, die zeit ziemblich kurzs, vnnd [in] dißer Zeit jederman mit jme selbs [bei sich zu Hause bleibt].

Biß vf des Früeling stillstand zu halten.

doch mag man es nicht destoweniger des volgenden Winter üben. Vnnd is schuelmeister seiner gehabens Müehe halben, es gange gleich für oder nit [es finde statt oder nicht], einer ergözlichheit vertröst worden. ${ }^{38}$

Alle diese Begriffe, Sterbent, Sterbeläufe, böse Luft und infizierte Luft, konnten aber noch nicht zu den obenerwähnten Maßnahmen führen: Maßnahmen gegen Bettler waren damals unter den verschiedensten Vorwänden auch sonst ergriffen 
worden; die Vorstellung von der infizierten Luft konnte allenfalls bewirken, daß es der einzelne vermied, sich solcher Luft auszusetzen. Wer sich aber nicht gänzlich, wie die Adeligen und Bürger von Rheinfelden, in sein Haus zurückziehen wollte, sondern eine gewisse Bewegungsfreiheit vorzog, dem blieb nur die Flucht übrig. Diese zwei Arten von individueller Prophylaxe waren allgemein anerkannt als die beste Möglichkeit, sich vor der Pest zu schützen.

Wie beschwerlich und umständlich sich eine Flucht gestaltete, wenn eine adelige Familie mit ihrem ganzen Hofstaat fliehen wollte, zeigt der Briefwechsel, den der Graf Georg von Thurn, Hauptmann und Landvogt des Oberelsaß ${ }^{39}, 1583$ mit der Stadt Rheinfelden führte: Wegen der Pest war er aus Ensisheim im Oberelsaß zuerst nach Wehr (nördlich von Säckingen), dann nach Säckingen geflohen. Nun fragte er in Rheinfelden an, ob es möglich wäre, daß er mit seinem ganzen Hof in Rheinfelden Aufnahme finden könnte, weil die Pest jetzt auch in der Nähe von Säckingen aufgetreten war. Und er erkundigte sich, ob die Stadt ihm unter anderm, gegen gebührende Bezahlung, die nötigen Gebäude, wie Wohnhaus und Stall, zur Verfügung stellen könnte:

«... dieweil sich die sterbeleuff zue. vnd vmb werr [Wehr] dermassen geneheret, das wier derowegen. vnd mehrer Sicherhait halben das negste orth $^{40}$. so darbei gewesen. fur die Handt genummen. vnd hieher gelangt, vnd wiewol es Got lob alhie noch gesund - so ist es doch als wier seithero vernummen an mehr orten nit weit von hinnen. auch nit zum Sichristen, derhalben wern wier fuernemblich bedacht. da wier zue Reinfelden mit Eurem Rath. Hilf beistandt. vnd furderung. neben besseren sicherheit. auch allerhandt mehrer gelegenheit zu Notturftigen hofwesen haben. vnd der gebuer noch zuuergnuegen versechen sein mechten, vnß aufs bäldist so jmmer muglich. auch derowegen zu Euch zueuerfuegen wann wier über Khurz. oder Lang. wie es Gott weiter schigkhen mecht. entweder widerumb weichen muesten. oder, da es besser zu Ensißhaim wurdt. zu negst dahin mit gelegenhait des Reinß zu wasser bequemer. Senfter. vnd geschwinder wegk Raisen khunten, Jst derhalben an Euch. vnser Freundlich vnd gnädig ansinnen. vnd begern, Jr wellet vnß zu Sunderer annemblicher wilfarung auf alle die mitl. vnd weg bedacht sein. vnß auch allerlei gelegenhaiten anzaigen, wie wier mit dangkhbarer vnserer bezahlung versechen sein mechten. vnd weder in einem. noch dem andern abgang noch mangel erliten. als mit allem dem. des fuernemblich in Kuchen vnd Keller. als dann auch im Stall fur vnßere pferdt. vnd sunst auch überall vnuermeidenlich gehabt muest werden, das alles Reiche vnß von Ewch vmb 
souil mehr dangkhbarer annemblichkheit, welches wier auch desto mehrers Jhe besser wier Euer auß Richtung fruchtbar befinden. vnd zum besten geniesßen werden, Sollichs zu Jeder furfallende gelegenheit desto dangkhbarlicher vmb Euch in allem freundlichen vnd gnädigen gueten willen zuuergleichen .... ${ }^{41}$

[dat. Säckingen, 5.3.1583]

Der Tägliche Rat, der dieses Gesuch behandelte, beschloß, dem Grafen abzuraten, nach Rheinfelden zu kommen:

«... Weil alhie keine leeren heüßer als das von Landeckhs seligen [...] vnd des von Berenfels gegen dem Kirchoff, da dann die gemein begräbnis ist, übergelegen dorab dann Jre gnaden ein abscheichen [Abscheu] holen möchten, zue dem der luft in etlichen der nechst vmb ligenden dörffern nit zum reinist sein woll $/ \ldots{ }^{42}$

Es mag verwundern, daß ein so hoher Regierungsbeamter wie der Landvogt des Oberelsaß ohne weiteres in dieser Gefahrenzeit seinen Regierungssitz verlassen konnte. Doch solange keine staatlichen Maßnahmen gegen die Pest ergriffen werden konnten, war es sogar vielleicht besser, wenn sich fähige Regierungsbeamte möglichst der Gefahr entzogen, um nach der Epidemie die politischen und sozialen Strukturen wieder neu zu organisieren.

Erstmals während des Pestjahres 1593 wurde in der Herrschaft Rheinfelden ein neues Konzept von Pest und Pestabwehr geschaffen. Der Oberamtmann Hans Othmar von und zu Schönau ${ }^{43}$ hatte nicht einfach die alten Vorstellungen über die Pestursachen übernommen, sondern hinzugefügt, daß, wie die Erfahrung zeige, die Pest auch «erblich» sei, was bedeutete, daß sie von Mensch zu Mensch, aber auch von infizierten Gegenständen auf den Menschen übertragbar sei. Da er aber die Vorstellung, das Gift könne sich auch durch die Luft ausbreiten, nicht einfach fallenließ, vermochte er durch die Verbindung beider Aspekte eine Grundlage zur Pestbekämpfung zu schaffen, die, wenn auch laienhaft ausgedrückt, der Kontagionslehre Fracastoros entsprach. Er postulierte also eine Übertragung durch Berührung, über Zwischenträger und auf Distanz. Daraus leitete er drei Gebote zur Verhütung einer weiteren Ausbreitung der Pest ab :

1. Niemand soll außer den mit der Pflege und Behandlung betrauten Personen («doctor, Scherrer vnd Bader») solche, die schon an der Pest erkrankt sind, besuchen.

2. Pestübertragende Gegenstände (Kleider und Betten) dürfen nicht aus infizierten Orten eingeführt werden. 
3. Niemand solle an Orte gehen, wo «diser vergift luft albereit eingerissen vnd nach einreissen möcht, ...». Aus solchen Orten dürfe auch niemand in noch verschonte Ortschaften mitgebracht werden.

Die vorgebrachte Analogie, daß ja auch beim Vieh gesunde und kranke Tiere getrennt gehalten werden, mußte auch den Untertanen Schönaus einleuchten; sie zeigt aber auch, durch welch anschauliches und unvoreingenommenes Denken der Oberamtmann zu seiner neuen Erkenntnis gekommen war. Sagte er doch selbst, daß es die eigene Erfahrung (und Beobachtung) gewesen sei, die ihn zu seinen Schlüssen geführt habe.

Schönau sah aber durchaus ein, daß es schwierig sein mochte, diese Befehle konsequent auszuführen: Daß man sich ohne echte Notwendigkeit («ohnne sondere ehehaften vnd notwendiche vrsachen ${ }^{44}$ ) nicht daran hielt, sollte zwar nicht gestattet werden; doch wer sich aus christlicher Nächstenliebe und andern triftigen Gründen verpflichtet fühlte, einen Angehörigen zu besuchen, dem konnte er dies nicht einfach verbieten. Hingegen drohte er, ähnliche Verfügungen zu treffen, wie sie in Ensisheim schon bestünden, falls jemand «mutwillig und vorsätzlich» seine Verbote nicht beachten würde. Ein solcher Übeltäter sollte aus seinem Wohnort ausgewiesen werden, oder dann müsse sein Haus verbarrikadiert werden. Beides sind Maßnahmen, die zeigen, daß in Ensisheim, der Hauptstadt Vorderösterreichs, die Pestprophylaxe schon weiter entwickelt war. Hier wurden nicht nur Kranke, sondern auch der Pest verdächtige Personen isoliert. Wie sich Ensisheim gegenüber verdächtigen Orten verhielt, geht leider aus dem Text nicht hervor. Jedenfalls scheint Schönau die «Suspension» (vgl. S. 209) noch nicht gekannt zu haben.

Auch Schönau sah in der Pest letztendlich eine Strafe Gottes. Offenbar konnte diese Vorstellung dazu führen, daß einige glaubten, man dürfe gegen das Schicksal, welches Gott über die Menschen in seinem Zorn verhängt hatte, nichts unternehmen. Gegen diesen an die calvinistische Prädestinationslehre erinnernden Glauben wendet sich Schönau mit einer interessanten These: Durch die Strafe Gottes sterben zu müssen, bedeutete für ihn, daß er ohne die Gnade Gottes ins Jenseits eingehen müßte. Deshalb, so folgert er, müsse man alles unternehmen, um nicht zu diesem Zeitpunkt, da Gott den Menschen zürnt, sterben zu müssen, denn es stehe einer gottähnlichen Kreatur viel besser an, in Gottes Gnade zu sterben, nachdem die Strafe vollzogen ist.

In seinem Befehl an die Vögte der Herrschaft streift Schönau noch kurz eine weitere - auch von den damaligen Ärzten anerkannte - Ansicht, daß jemand, der einen Kranken besuche, darob dermaßen erschrecken könne, daß er bald darauf selbst von der Pest ergriffen werde. 
Um Plünderungen von ausgestorbenen Häusern vorzubeugen, aber auch damit keine infizierten Gegenstände vorzeitig wiederverwendet würden, befiehlt er den Amtleuten der drei Landschaften, alles zu versiegeln und die Schlüssel des betreffenden Hauses an sich zu nehmen, bis sich die Luft wieder gebessert habe.

Dieses aufschlußreiche Schreiben soll im folgenden ungekürzt wiedergegeben werden:

«An die Oberuögt vnd Andern

Amptleüth des Möhlin bachs,

Vnnsern grues vnd fründtlich dienst zuuor, Ersamen lieben vnd guete fründt, demnach der böß vergiftisch pestilenzisch luft, etlicher orten, vnnserer benachparten, Alls zu wer [Wehr], hasell [Hasel] vnd jn diser vnserer gnedigist Anbeuolhenen, Heerschaft Rheinfelden, zu Münchweilen, vor gueter zeit eingerissen, vnd wie man mit Mumpf, vnseren auch getrewen lieben Ampts Ewern mit vnderthannen, auch Öfflichen vnd Seckhingen laider exempell genug vnd in täglicher erfarung hat, das solche sucht leichtlich von einem orth, an das ander durch das stetig ohn abscheüchig zusamen wandern, ererbt vnd gepracht würdet, dan auch wir Ewere fürgesetzte nehere Obrigkheit, für der vnderthanen leiben, haab vnd guet, soueil an vns, guet sorg vnd achtung zu haben auch vor vngefähl zuuerhieten, schuldig seyen, Alls ist an eüch sambt vnd sonders, vnser Amptlicher beuelch, auch güetlichs verwarnen vnd begeren,

Weil man doch zwischen den gesunden vnd Kranckhen viech ein vnderscheidt halte, Veil mehr es mit dem Menschen alls der Edlisten vnd Gott gleich erschaffenen Creatur beschechen solle, vnd aber veil pesser in der gnaden, dan straf gottes (, wie dan dise sucht ein straf ist,) zu sterben, jr wellent den vnderthanen das vnser woll meinendts jnen zum pesten Angestehen, schreiben fürhalten vnd beuelhen, das sie sich soueil die Christenliche lieb vnd gebeür erleiden mag, schonen vnd abhalten, an die jhenigen orth vnd Endt, diser vergift luft albereit eingerissen vnd nach einreissen möcht, zu ghan, oder von selbigen orthen, Kleider, Beth, oder dergleichen, dahero es gar baldt zu ererben, an dz Ander zu bringen. auch den zu wandel des Volckhs von vngesunden orthen, mit nichten zu gestatten, dan was were dem Kranckhen mit dem jhenigen der jme besucht, vnd jme doch nit helffen khan, oder hingegen dem der einen Andern in der Kranckheit besucht, der erschrickht, kranckh würdet den vnfahl ins hauß bringt oder Villeicht mit weib Khindt, vndt gesindt gar stirbt vnd der Kranckh den Er erstens besuecht hat khombt so baldt wider auf damit gedient vnnsers 
erachtens gar nichts dan zu solchen Kranckhen allein doctor, Scherrer vnd Bader, die für solche suchten jre gebeürende mitl zu gebrauchen wissen, auch leüth die den Kranckhen nodtturftiger wart, vnd pfleg thuen Khönden, gehoren, versehent vns derohalben, jr die Amptleüth selbs, so woll auch die vnderthanen, werden sich auf dise vnnsere getrewe herzige Vatterliche verwarnung vnd angestellte Ordnung aller gebeürenden gehorsamen, vnd bescheidenheit hierunder erhalten vnd sich allso vnder vnd gegen einandern massen das man von Obrigkheit vnd Ampts wegen nit vrsach habe, Jhemanden der Also mutwillig vnd fürsezlich ohnne sondere ehehaften vnd notwendiche vrsachen, an ein vnd dz ander vngesundt Orth lauffen. oder von selbigen orthen hero leüth einziechen wurde, den Fleckhen zuuerbieten, oder jre Heüser zue zeschlagen, Alls zu Enßißheim vnd anderswo mehr beschickht,

damit dan Niemandt sein gebeürendt Erbschaft, die jme an einem oder anderen Orthen zufallen möcht, entzogen werde, So ist hiermit vnnserer beuelch, das allßbaldt ein Par eheuolkh im Hauß sturbe, das allßbaldt der Amptman im selben Fleckhen, sambt einem oder zweyen geschwornen, alle sachen einem jeden zu seinem rechten, vorsorgen, einbeschliessen, die trög ver Pitschieren, vnd der Amptman die Schlüssell beyhanden behalten sollen, biß gott wider friden vnd Pessern luft schikht, wolten wir Eüch zu einem wissen vnd nachrichten nit verhalten, Was vnd eüch hiemit dem Allmechtigen, in seinem gnadenreichen segen vnd glückhlicher gesundtheit threwlich beuelchende,

dat Rheinfelden den 14. Augustj, Ao $1593^{43}$.

$$
\begin{aligned}
& \text { Schonaw, } \\
& \text { Pirr }{ }^{45}
\end{aligned}
$$

An den Vogt zu Mumpf, wo ja die Pest schon ausgebrochen war, sandte Schönau noch am selbigen Tag ein Sonderschreiben. Dieser Vogt, Ulrich Boni («Vllin Bonnj») führte in Mumpf eine Gaststätte, die von Fuhrleuten und Gästen jeden Standes besucht wurde. Dem Text nach ist nicht auszuschließen, daß die Pest hier zuerst ausgebrochen war. Jedenfalls hatte Boni in seiner Familie schon einen Todesfall zu verzeichnen. Dennoch hatte er zum Ärger Schönaus nichts aus dem «leidigen grosen vnfahl» gelernt, ja er hatte sogar seinen Stiefsohn Kaspar Dinkel («Caspar dinckhel») genötigt, in seinem Hause zu verbleiben, anstatt ihn unverzüglich von der Herberge fortzubringen und diese zu schließen.

Die Herberge war auch sonst ein Stein des Anstoßes für Schönau: Als Treffpunkt von Fuhrleuten und als Übernachtungsgelegenheit für zweifelhafte fremde 
Personen auf der Durchreise war sie geeignet, die Pest weiter zu verstreuen. Damit dies nicht geschehen könne und damit ehrliche Adelige und andere gute Leute, die mit diesen Verhältnissen nicht vertraut waren, nicht gefährdet würden, befahl Schönau dem Wirt und Vogt, seine Herberge bis auf weiteres zu schließen. Dann gibt er ihm in Kürze dieselben Anweisungen, die er den Amtsleuten in seinem Rundschreiben gemacht hatte:

«An Vogt zu Mumpf Vllin

Bonnj,

Vnseren grues vnd alles guets zuuor, lieber Vogt, wir tragen Eüwerer traurigen zuständt, vnd begägneten vngefähl mit eüch ein Christenliches bedauren vnd mit leiden, der Allmechtig wölle allen Christgleübigen Seelen gnedig sein, seinen Zorn miltern, vnd wider fürderlichen pessern luft verleichen,

Hetten vnns aber zwar zu eüch einer mehrern bescheidenheit getröstet, Alls da jr den leidigen grosen vnfahl im Hauß gesehen, vnd mit ewerm schaden erfahren wie leichtlich solche sucht zu erben, das jr (.wie wir bericht.) Eürem Stief-Sohn Caspar dinckhel vfhalten, vnd bey eüch jm Hauß zwar zuuer pleiben nötigen wellen, sonder were eüch Löblicher vnd besser angestanden, das jr selbs den Knaben an sicher Orth verschaft, vnd Ewer Herrberg vngeacht des zeitlichen nuzes zugeschlagen, niemandts mehr beherbergt hetten, dardurch niemanden in gfohr vnd nachtheill khomen were, weil aber von eüch durch die Fuohrleüth auch ander hochen vnd nider standts Personen, das wider spyll [Widerspiel = Gegenteil] [getan wird] vnd soueil fürkhombt, das jr eüch von frembden, durch reisenden Personen nit abhalten, sondern Eüch verdrüßt, wan sie euch scheüchen, ists Eüwerthalb desto schimpflicher zue hören,

damit dan nit Also vnwüssender dingen etwan ehrliche Adels personen, oder andere guete leüth, bey eüch einziechen, etwan ererben, vnd in gfohr khommen, auch den bössen luft an andere Orth bringen mochten, desen wir hernach verwiß zue gewerten, So wellent des schilt herab thun vnd ein Zeit lang biß auf bessere gelegenheit vfhören würten, dan sonsten die herrberg da ein Gast darinen Kranckh werden solt, veil mehr vnd weiterer verschreit wurde, ...» ${ }^{46}$

Eine sinnvolle Pestprophylaxe, wie sie Schönau gern verwirklicht hätte, ist nur dann möglich, wenn die Maßnahmen sich einheitlich auf ein größeres Gebiet erstrecken, welches politisch, vor allem aber auch wirtschaftlich ein in sich geschlossenes Gebilde darstellt. Dies war zur Zeit Schönaus keineswegs der Fall. 
Wirtschaftlich gehörte die Herrschaft Rheinfelden zum Einzugsgebiet der «Großstadt» Basel, während die kleine Stadt Rheinfelden nur eine untergeordnete Rolle spielte. Zudem hatte Schönau, wie es schon in der vorangehenden Epidemie von 1582 zum Ausdruck kam, nur eine beratende Funktion inne; die Stadträte hatten damals gegen das Begehren Schönaus den Jahrmarkt abgehalten. Damit war das Aktionsfeld Schönaus stark eingeschränkt. Eine weitere Komplikation ergab sich durch den Umstand, daß Schönau selbst in der Stadt Rheinfelden wohnte: Als Oberamtmann der Herrschaft Rheinfelden war er aber darauf angewiesen, mit seinen Untertanen in Kontakt zu bleiben, auch wenn in einem Dorf, wie zum Beispiel in Mumpf, die Pest ausgebrochen war. Trotzdem war er sehr darum besorgt, daß die Pest nicht in die Stadt eingeschleppt wurde, in der er seinen Wohnsitz hatte. Kamen die Leute aus infizierten Dörfern der Herrschaft in die Stadt, bestand nach Schönau die Gefahr, daß Schultheiß und Rat Befehl geben würden, niemanden mehr von dort in die Stadt einzulassen, so daß der Kontakt zwischen Schönau und der Herrschaft abgebrochen würde. Diese ambivalente Situation bewog wahrscheinlich Schönau, den folgenden Satz an den Vogt zu Mumpf nicht schärfer und deutlicher zu formulieren:

«..., So sollen jr Ewerer Gemeindt beineben, anzeigen, das sie sich mit dem zu lauff an gesunde Orth sonderlich alhie in die Stat Rheinfelden so veil müglich wie auch jr selbs vndt Ewer gesindt etwas massen, vnd vnser darunder verschonnen wöllen, damit den Herrn der Stat nit vrsach geben werde, Niemanden von Mumpf mehr ein zue lassen, ... ${ }^{47}$

Doch vorläufig waren die «Herrn der Stat» noch weit davon entfernt, solch rigorose Maßnahmen zu treffen. Aber auch Schönaus Amtsnachfolger, Johann Jakob Eggs, hatte während der darauffolgenden Epidemie von 1609-1611 die gleichen Befürchtungen. In einem Mandat, das im übrigen fast wörtlich die Anweisungen Schönaus wiederholt, wies er deshalb schon im voraus die Ober- und Untervögte an, daß seine Untertanen nur noch durch unverdächtige Mittelspersonen mit ihm verkehren sollten [29. November 1610], falls die Stadt tatsächlich niemanden mehr aus infizierten Orten einlassen würde:

«Wann dann auch in alhiesiger Statt. die ordnung gemacht were das man von den Jhenigen orten. da dergleichen böse luft eingerissen. niemandt mher einzulassen bedacht. So wöllen wir Euch dessen hiemit berichtet vnd darbey weiter beuolhen haben. solches zugleich bey einer straff zuuerbietten. dass vß keinem ortt. alwa die böse sucht regiert. niemandts mher hieher komme. Jr aber auch. damit einem Jeden dennocht sein anligen der gebeür verricht werden möchte, ein Person verordnen vnd 
besteln sollen, der dieselben anhöre, dennach derselb da es vonnöten. solches dem [?] Wölfflin dem Würt in der Auw oder dem Oberuogt anbringen. vnd fernnern beschaidts darüber erwarte. oder Jr die Vögt dasselbig selbs also verrichten Es sollen aber vff solchen fahl. weder Jr die Vögt. noch die Jhenigen. so Jr hierzu verordnen. an khein sterbenden ordt. bey hoher straff. nicht gehen. sonder selbsten schonen ... ${ }^{48}$

Offenbar noch am selben Tag verordneten die Rheinfelder Räte dies für die Gemeinden Degerfelden in der Landschaft Rheintal ${ }^{49}$. Daß nun auch die Stadt Rheinfelden strengste Maßnahmen verordnete, ist weniger den Stadträten als vielmehr dem Eingreifen der vorderösterreichischen Regierung in Ensisheim zuzuschreiben. Noch zwei Monate zuvor, am 21. September 1610, hatten die Stadträte, nach einem Städtetag in Laufenburg, eine Bürgergemeinde einberufen, und obwohl bekannt war, daß in Basel die Pest herrschte, ließen sie es dennoch zu, daß die Bürger, falls sie eine Sondererlaubnis des Schultheißen erhielten, nach Basel gehen durften:

«Sollen die burger auch jr weib, khindt vnd gesündt, die Stat Basel vnd andere inficierte orth soueil mensch vnd müglich meiden, vnd ohne erlaubtnus des herren Schultheißen vnd Stathalter, auch ohnne sonderbare wichtige vrsachen, der endts nit ziechen, ...» ${ }^{50}$

Aber auch für die Einwohner der Stadt Basel wurde noch kein absolutes Verbot aufgestellt, nach Rheinfelden zu kommen, der Tägliche Rat bestimmte nur:

«Der Statt Basel, Nachperlichen zu schreiben, jre burger souil müglich Abzumanen, sich des Jarmarckhts zu enthalten. » ${ }^{51}$

Wer allerdings ohne Erlaubnis nach Basel ging, wurde bestraft. So wurde «Vlrich Webern, genent Aufrechten, fürgestelt, vnd jme mit ernst verwiesen, das er zu Basel gewesen vnd gesagt habenn soll, was er dem Schultheißen vnd seinen gebottenn nachfrage, der vrsachen jn Thurn erkhanndt, doch solle der sachen bey Hannß Bernhardt bürge besser nachgefragt werden.$/$. $^{52}$

Vier Tage später wurde im Großen Rat das soeben eingetroffene Schreiben der Vorderösterreichischen Regierung und Kammer behandelt, welches zu schärferen Maßnahmen, aber auch anderen Neuerungen führte. Tags darauf, am Sonntag vor Galli [16. Oktober 1610], wurde es

«... jm Buchstaben abgelesenn, mit dem anhang, demselben zu gehorsamen auch niemanden der von inficierten orthen herkombt vnderschlauff zu geben, oder dieselben einzuziechen [in die Stadt mitzubringen], Item 
die burger so khind zu Basel oder anderst wa haben, dieselben nit zu besuchen, vil weniger daheimben zu fordern, wer darwider thun wurde, sol mit ernst gestraft oder ein zeit lang als ein monath der Statt verwisen werden. $»^{53}$

Damit wurde in der Stadt Rheinfelden eine Art Quarantäne eingeführt. Es war dies eine Maßnahme, welche von Politikern vorgeschlagen und durchgesetzt wurde. Von Medizinalpersonen wurde dazu kein Beitrag geleistet. Im Gegenteil, die Bader mußten zweimal kurz hintereinander gemahnt werden, niemanden mehr aus infizierten Orten in ihren Badestuben anzunehmen:

«Die Bader verwarnet, diejhenigen so von Lörach vnd Riehen alher jn die Badtstuben khomenn möchten, abzuweisen.» [21. September 1610] ${ }^{54}$

«... Die Bader furgestelt, vnd ihnen wahrnungsweiß vndersagt, die von inficierten orthen, nit zur Badstuben khomen zu lassen.»

(Zinstag vor Galli [16. Oktober 1610]) $)^{55}$

Die einzige ärztliche Beratung, die die Rheinfelder Behörden jemals während einer Pestepidemie erhielten, ist insofern enttäuschend, als sie offenbar von einem Anhänger der reinen Miasmalehre stammte: Der recht berühmte Leibmedicus des Erzherzogs Leopold, Dr. Friedrich Eggs ${ }^{56}$, der selbst aus Rheinfelden gebürtig war, empfahl in einem Schreiben an seine Vaterstadt nur,

«... wegen der laider vor augen schwebenden sterben leüffen, vnd des jn der Stat hin vnd wider wesenden vnraths [...], denselben soueil müglich abzuschaffen, die Brunen sauber zu halten, vnd den Bach jn der wochen etliche male jn die Statt Lauffen zu lassen. » [30. November 1610] ${ }^{57}$

Sechs Tage später wurde die Mahnung an die Gemeinde weitergegeben. Die Ausführlichkeit, mit der dies geschah, zeigt, daß die Räte diese allgemeinen Hygienemaßnahmen, welche dazu dienen sollten, die Luft rein zu halten, mindestens ebenso wichtig nahmen wie die Sperrmaßnahmen, die ihnen von der vorderösterreichischen Regierung auferlegt worden waren. Auch waren die neuen Sperrmaßnahmen weit weniger gut kontrollierbar und stellten ein neues organisatorisches Problem dar. Die Brunnenreinigung war aber auch schon früher und außerhalb der Pestzeit eine Notwendigkeit. Hier nun der ausführliche Text für die Bürgergemeinde:

«... vnd weil durch vnrath vnd reuerenten $[=$ indem über das Schreiben von Dr. Eggs referiert wird ? vgl. S. 212 infiziert = jnuiciert] zu melden, Müstheüffen in solchen seüchten grossen vnrath endtstet, Sol man denselben jn den gemeinen strassen vnd gassen sonderlich, als teglichen kirch- 
gang bruchen mueß, bey straff $1 . £$. [Pfund] stebler ${ }^{58}$ jnerhalben 14. tagen, auß der Stat füeren, vnd über ein Pahr kerren [paar Karren] voll nit mehr zusammen kommen lassen, sondern denselben jheweils abfüeren, wer derwider handelt, sol nit allein obgesetzte straff abstatten, sondern der Paw Preiß ${ }^{59}$ gegeben werden.

... weil abermalen augenscheinlich, das die gemeinen Prunen gar verwüst vnd vnsauber gehalten werden, vnd aber solches nit zu gestatten So wil man die burger des allen nochmalen alles ernsts erjndert, und jnen benebens beuolheen haben solches bey jren weibern, döchtern vnd gesündt abzuschaffen vnd zu beuelhen, nichts vnsubers jn die Prunen zu stossen, oder zu schütten, deßgleichen khein wuest oder dergleichen in Statbach zu werffen wer es übersicht, sol jederweilen darumben abgestraft werden $\ldots)^{60}$

Es erstaunt, daß Rheinfelden offenbar von den ätiologischen Vorstellungen des Felix Platter (1536-1614), des Stadtarztes im benachbarten Basel, nichts übernommen hatte. Er hatte die Miasmalehre verworfen und ein Pestgift angenommen, das sowohl durch Berührung als auch durch die Luft übertragen werden konnte. Es war nach Platter von Anbeginn der Welt in bestimmten Körpern und befiel von Zeit zu Zeit die Menschen ${ }^{61}$.

Erst im Januar 1611, als es ernst galt, eine Ausbreitung der Pest innerhalb der Stadt zu vermeiden (das Totenregister ${ }^{62}$ zeigt seit Beginn des Monats November 1610 eine um das Sechsfache erhöhte Mortalität), bestimmte der Große Rat am 13. Januar 1611 erstmals, den Jahrmarkt «fürgefalner vrsachen vnd bewegender bedenckhen halb, abzustellen vnd abzuschreiben ». [RP, 13. Januar 1611] ${ }^{63}$ Dies geschah, damit weniger Gefahr war, daß Infizierte und Gesunde an einem Ort zusammenkamen.

Erst während der Epidemie von 1628-1630 wurde der Jahrmarkt verboten, um ein Einschleppen in die Stadt von außen her zu vermeiden:

«Jarmarckhts halber. wegen aller vmbgrenzenden Orthen Leider ziemblich starckh grassierender Contagion dißmalen abzustellen vnd zue vnderlassen.» [Beschluß des Großen Rates vom 24. August 1629]

Es ist sicher nicht von ungefähr, daß hier zum erstenmal das Wort «contagion» für Pest gesetzt wurde. In der Folgezeit bürgerte sich der Ausdruck dermaßen ein, daß andere Begriffe für die Pest kaum mehr angewendet wurden. Damit war in Rheinfelden eine neue Ära der Pestbekämpfung eingeleitet worden. Grundlage dazu war das Akzeptieren der Kontagionslehre im Sinne Fracastoros, die 83 Jahre zuvor von dem Veroneser Gelehrten aufgestellt worden war. 
Zusammenfassend läßt sich sagen:

Eine chronologische Gegenüberstellung der ätiologischen Vorstellungen und politischen Strukturen einerseits und der ergriffenen prophylaktischen Maßnahmen anderseits ergibt für die Stadt und Herrschaft Rheinfelden des 16. und 17. Jahrhunderts :

- Daß eine Prophylaxe nur betrieben wurde, wenn ätiologische Vorstellungen diese rechtfertigten.

- Die aus der Antike übernommene Miasma-Lehre (Lehre von der Luftvergiftung) führte zu allgemeinen Maßnahmen der öffentlichen Hygiene.

- Die Übernahme der Kontagionslehre ermöglichte im Laufe des 17. Jahrhunderts die Entwicklung spezifischer prophylaktischer Maßnahmen gegen die Pest:

Gegen außen Bando und Suspension (die die Einreise oder Durchreise von Personen und die Einfuhr bzw. den Transit von Handelsgütern regelten, vgl. S. 209).

Im Innern die Isolation der Kranken.

- Damit die Maßnahmen ihre Wirkung hatten, mußten sie sich über ein möglichst großes Gebiet erstrecken, das politisch und wirtschaftlich eine Einheit bildete.

- Durch diese Faktoren verlor die individuelle Prophylaxe gegenüber den öffentlichen, von den Behörden angeordneten Maßnahmen an Gewicht.

Anmerkungen, Quellennachweis

Abkürzungen :

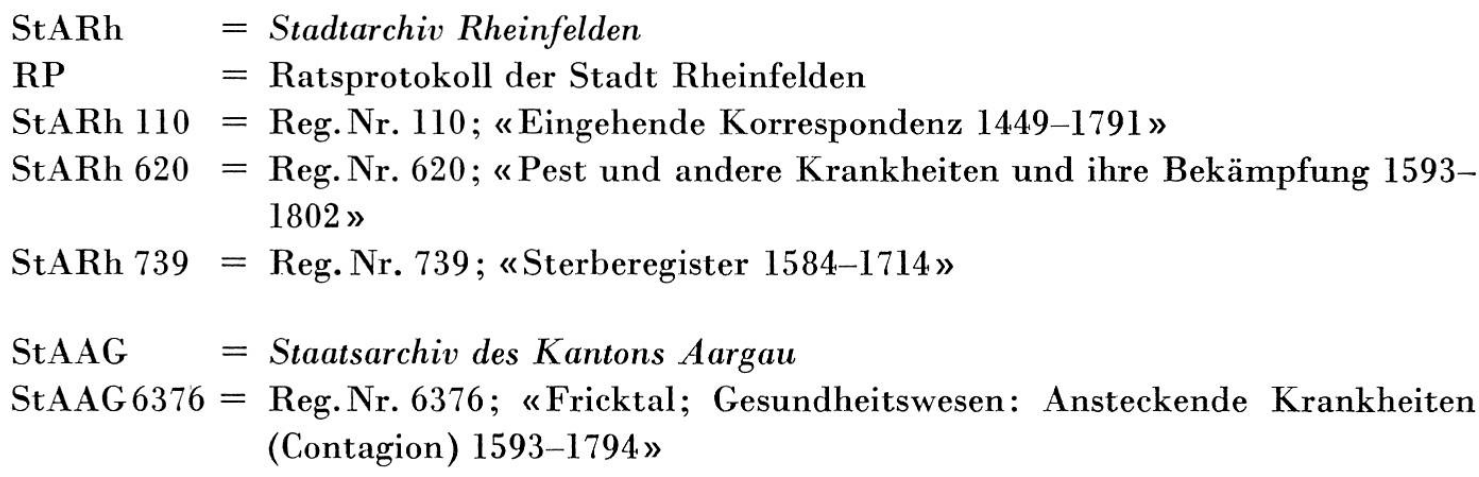

Die Quellentexte aus dem Stadtarchiv Rheinfelden und dem Staatsarchiv des Kantons Aargau wurden originalgetreu transkribiert. Eigene Einschübe stehen in [ ]; Unleserliches wurde durch [?] ersetzt. 
${ }^{1}$ Schib, Karl, Die vier Waldstädte. In: Vorderösterreich, eine geschichtliche Landeskunde, hrsg. von Friedrich Metz, 2.Aufl, Freiburg i. Br. 1967 (= Schib, Waldstädte), S. 383.

2 Schib, Waldstädte, op. cit., S. 384.

3 Schib, Waldstädte, op. cit., S. 383.

4 Schib, Waldstädte, op. cit., S. 385.

5 Schib, a.a. O.

${ }^{6}$ Schib, Karl, Geschichte der Stadt Rheinfelden, Thayngen-Schaffhausen 1961 (= Schib, Rheinfelden), S. 83-88.

7 Oft war dieser Oberamtmann auch Vogt der vier Waldstädte. Vgl. Senti, Anton, Die Herrschaften Rheinfelden und Laufenburg (in: Vorderösterreich, op. cit.), S. 409.

${ }^{8}$ Senti, A., op. cit., S. 429.

${ }^{9}$ Senti, A., op. cit., S. 409.

Zur Geschichte von Stadt und Herrschaft Rheinfelden sowie zu Vorderösterreich habe ich noch folgende Literatur konsultiert:

Burkart, Sebastian, Geschichte der Stadt Rheinfelden, Aarau 1909.

Bröchin, Ernst, Kulturhistorische Rheinfelder Chronik, Rheinfelden 1944.

[Kreutter, Franz], Geschichte der K. K. Vorderösterreichischen Staaten, 2 Bde., St. Blasi 1790.

Stolz, Otto, Geschichtliche Beschreibung der ober- und vorderösterreichischen Lande (Quellen und Forschungen zur Siedlungs- und Volkstumsgeschichte der Oberrheinlande, Bd. 4), Karlsruhe 1943.

10 Fritz Glauser zeigte, daß der Gotthardverkehr im letzten Viertel des 16. Jahrhunderts stark zurückging, weil «südlich der Alpen offenbar eine eigentliche Pestpsychose entstand». Auf Kosten des Gotthardverkehrs nahm der Churer Transit großen Aufschwung. Glauser, Fritz, Der Gotthardtransit von 1500 bis 1600 (Numéro spécial: Histoire des Alpes. Perspectives nouvelles, hrsg. von Jean-François Bergier). In: Schweizerische Zeitschrift für Geschichte 29 (1979), S. 38-40.

11 Glauser, F., op. cit., S. 18-22.

12 Die Schweiz (Bibliothek des Geographischen Lexikons der Schweiz; Red. Heinrich Brunner), Neuenburg 1909, S. $82 \mathrm{f}$.

13 Stolz, O., op. cit., S. 161.

14 Glauser, F., op. cit., S. 18-22.

15 Stolz, O., op. cit., S. 82, $93 \mathrm{f}$.

${ }^{16}$ Zur Entstehung der Zurzacher Messen: s. Attenhofer, Edward, Alt-Zurzach, Aarau 1940, S. 35-40.

17 Nach Senti, A., op. cit., S. 405, und Attinger, E., op. cit., S. 39, abgeändert.

${ }^{18}$ Hug, Johann Christoph, seit 1648 Oberamtmann der Herrschaft Rheinfelden, wurde 1663 vorderösterreichischer Regierungsrat und 1669 von Kaiser Leopold I. geadelt (Hug von Hugenfeld).

19 Vgl. hiezu die Akten Fricktal, Reg. Nr. 6376 g. Gesundheitswesen: Ansteckende Krankheiten (Contagion) 1593-1794, im Staatsarchiv des Kantons Aargau (StAAG 6376).

${ }^{20}$ Bando, Suspension: Eine genaue Definition dieser Begriffe findet sich in einem Schreiben des Sanitätsmagistrats von Venedig an Bürgermeister und Rat der Stadt Basel, dat. 
vom 14.Juli 1715, welches Basel in deutscher Übersetzung an den Baron Ignaz von Grammont, Obervogt der Herrschaft Rheinfelden, weitersandte (StAAG 6376):

«... dergestalten, daß allen und jeden, wer die auch sind, immer und zu alles zeiten, und das bey den schwärsten Straafen, auch der Lebens Straaf verbotten seye und bleiben solle. Weder Persohn, Vieh noch Waaren auß denen durch gegenwärtiges Mandat Bandisierten Landen in den Venetianischen Stand zu bringen noch zu introducieren, noch auch zu dero Jntroducierung oder Einführung behülflich zu seyn, im übrigen sollen die auß denen samtlichen suspendirten und in disem Mandat ernambsten Landen kommende Persohnen und Waaren, in den ofentlichen Lazarethen die erforderliche Außreinigung der Contumaz für so lang außhalten, als ihnen vorgeschriben wird.»

${ }^{21}$ Sticker, Georg, Abhandlungen aus der Seuchengeschichte und Seuchenlehre, I. Bd.: Die Pest, 2. Tl., S. 13.

${ }^{22}$ Stettler, Antoinette, Der ärztliche Pestbegriff in historischer Sicht. In: Gesnerus 36 (1979), Heft 1/2, S. 127.

${ }^{23}$ Koelbing, Huldrych M., Diagnose und Ätiologie der Pest bei Felix Platter (1536-1614). In: Medizinische Diagnostik in Geschichte und Gegenwart, Festschrift für Heinz Goerke, München 1978, S. 224.

${ }^{24}$ Schib, Rheinfelden, op. cit. sub 6, S. 300.

${ }^{25}$ Für die Stadt Rheinfelden finden wir in der Sekundärliteratur folgende Pestjahre (bis Mitte des 16. Jahrhunderts): 1146 (B), 1349 (S), ca. 1420 (S), 1439 (B), 1541 (B, S), 1552 (B).

$\mathrm{B}=$ Burkart, S. $347 ; \mathrm{S}=$ Schib, S. 300.

${ }^{26}$ StARh, RP Zinnstag nach Verenae [1. September] 1564.

${ }^{27}$ StARh, RP Zinstags post Simonis \& Judae [28. Oktober] 1582.

${ }^{28}$ Biraben, Jean-Noël, Les hommes et la peste, Bd. 2, Mouton 1976, S. 9.

${ }^{29}$ Schönau, von und zu, Hans Othmar (1559-1608), seit 1580 Hauptmann der vier Waldstädte und Oberamtmann der Herrschaften Rheinfelden und Laufenburg.

30 Vngelter = Steuereinzieher (Schweizerisches Idiotikon, Bd. 2, Frauenfeld 1885, S.244, Sp. 2.

${ }^{31}$ StARh RP, Freitags vff Amandi [26. Oktober] 1582.

${ }^{32}$ StARh RP, [28. Oktober] 1582.

${ }^{33}$ StARh RP, Zinstag nach Martini [11. November] 1582.

${ }^{34}$ Die Stadt Rheinfelden hatte drei Zünfte: Zunft zum Bock, zum Gilgenberg und Kaufleutezunft. (Schib, Rheinfelden, S. $81 \mathrm{f}$.)

${ }^{35}$ StARh RP [28. Oktober] 1582.

${ }^{36}$ StARh RP vigilia Nativitatis Maria [7.September] 1593.

${ }^{37}$ Burkart, S., op. cit., S. 710.

${ }^{38}$ StARh RP, Zinstag vigilia Nativitatis Maria [7.September] 1593.

${ }^{39}$ Georg von Thurn, Graf zu Valsassina [Tal südlich des Veltlins; es gehörte damals zum Herzogtum Mailand] und Freiherr zum Kreuz [Kreuzburg im Oberelsaß?], geheimer Kammerrat des Erzherzogs Ferdinand zu Österreich, Oberst, Hauptmann und Landvogt des Oberelsaß.

40 Mit «negste orth» ist Säckingen gemeint.

${ }^{41}$ StARh $110^{3}$.

42 StARh RP, Zinstag nach Oculj [20. März] 1583; vgl. auch Konzept des Stadtschreibers: 
«Dem Wolgebornen Herrn,

Geörgen Grauen von Thurn

ze Valsasina vnd zum Creütz/

der F: dt: Erzherzog Ferdinand zu

Osterreich/geheimen RathCammer/

Oberst/ten/ Hauptman vnd Landuogten

Obern Elsaß ...», dat. 6. März 1583 (StARh $\left.110^{3}\right)$.

43 vgl. Anm. 29.

44 ehehaft $=$ echte Not (Der große Brockhaus, Leipzig 1930). Das Zitat stellt also einen Pleonasmus dar.

${ }^{45}$ Pirr, Michael, Einnehmer der Herrschaft Rheinfelden.

46 StARh 620.

47 StAAG 6376.

48 Johann Jakob Eggs, lic. iur., seit 1596 Oberamtmann und v. ö. Rat.

49 StAAG 6376.

50 RP, Samstags vor Mathei apostoli [21.September 1610].

${ }^{51}$ RP Zinstag vor Galli [16. Oktober 1610].

52 ibid.

${ }^{53}$ RP [16. Oktober 1610].

${ }^{54} \mathrm{RP}$, vff Mathei apostoli ein Gemeindt vnd den burgern fürgehalten.

${ }_{55}$ RP [16. Oktober 1610].

56 Friedrich Eggs (1572-1638) studierte in Freiburg i. Br. und Ingolstadt Philosophie und Medizin. Nach weiteren Studien in Löwen bei Johann Baptist van Helmont doktorierte er in Padua. Erzherzog Leopold von Österreich ernannte ihn zu seinem Leibmedicus. Er starb 1638 in Graz.

57 RP, Freitags nach Andreae apostoli 1610.

${ }^{58}$ Stebler $=$ geringhaltige silberne Pfennigmünze (Schweizerisches Idiotikon, Bd. 10, Frauenfeld 1939, Sp. 1060.

59 Paw Preiß, Paw = Mist (Schweizerisches Idiotikon, Bd. 4, Frauenfeld 1901, Sp. 19481950.

${ }^{60} \mathrm{RP}$, Sct Nicolai episcopi, gebot vff den Zünften vnd den burgern fürgehalten wie volgt. [6. Dezember 1610]

${ }^{61}$ Koelbing, H.M., op. cit., S. 222 f.

${ }^{62}$ StARh 739, Sterberegister 1584-1714.

${ }^{63}$ RP, Freitag nach Hilarij [13. Januar 1611].

\section{Verdankungen}

Herrn Professor Koelbing möchte ich an dieser Stelle für seine mannigfaltigen Bemühungen und Ratschläge meinen ganz besonderen und herzlichen Dank aussprechen. Danken möchte ich auch Herrn Dr. Jean Jacques Siegrist, Staatsarchivar des Kantons Aargau, der mir die Archiv-Verhältnisse im Aargau aufzeigte und historische Hinweise gab, sowie Herrn Haudenschild und den Angestellten der Stadtkanzlei Rheinfelden, die mir die Benützung der Archive ermöglicht haben. 


\section{Summary}

The author discusses the influence of aetiological concepts and political structures on plague prevention in the town and territory of Rheinfelden (Austrian until 1798, now Swiss). Here as elswhere, mercantile interests often prevented strong action, especially rigorous suspension of commerce with infected places.

In the 16 th century, the theory, inherited from Antiquity, that plague was caused by corrupted air (miasma) still prevailed; it stimulated the town authorities to introduce general sanitary measures. In the 17 th century, the political authorities-the Council of the town of Rheinfelden as well as the Governor of the surrounding territory-acted more and more on the principle that plague is a contagious disease; strict control of travel and trade was now enforced. The measures applied were the same that had been developed centuries ago already by the Italian cities («bando», «suspension»). In order to prevent the spread of the "contagion» in the town itself, infected persons were isolated in their houses.

With this change of prevailing aetiological concepts, personal prophylaxis (including flight from a plague-ridden place) lost its importance in favour of prevention on a larger scale by administrative public measures.

Dr.med.Vera Waldis

Medizinhistorisches Institut

der Universität Zürich

Rämistraße 71

8006 Zürich 